\title{
Effectiveness, Adherence, and Safety of Evolocumab in a Swiss Multicenter Prospective Observational Study
}

\author{
David Nanchen (1) - David Carballo (1) - Stefan Bilz (1) · \\ Hans Rickli (1) - Konstantinos C. Koskinas - François Mach (D) · \\ Christian Mueller (1) - Carmela Crljenica (1) - Mariagrazia Rossi (1) · \\ Nina Reichert (1) · Isabella Sudano
}

Received: September 9, 2021 / Accepted: October 12, 2021 / Published online: November 18, 2021

(c) The Author(s) 2021

\begin{abstract}
Introduction: The aims of this study were to describe patient characteristics, lipid parameters, lipid-lowering drug use, and safety of patients receiving evolocumab in a real-world clinical setting.

Methods: We conducted a 1-year multicenter observational study of adults using evolocumab with confirmed atherosclerotic cardiovascular disease (CVD) or at high cardiovascular risk, and elevated LDL-C despite maximally tolerated
\end{abstract}

Supplementary Information The online version contains supplementary material available at https:// doi.org/10.1007/s12325-021-01962-w.

D. Nanchen $(\bowtie)$

Center for Primary Care and Public Health,

University of Lausanne, Lausanne, Switzerland

e-mail: david.nanchen@unisante.ch

D. Carballo · F. Mach

Division of Cardiology, Department of Medicine,

Geneva University Hospital, Geneva, Switzerland

S. Bilz

Division of Endocrinology and Diabetes,

Kantonsspital St. Gallen, St. Gallen, Switzerland

H. Rickli

Cardiology Division, Kantonsspital St. Gallen, St.

Gallen, Switzerland

K. C. Koskinas

Department of Cardiology, Bern University

Hospital, Inselspital, University of Bern, Bern,

Switzerland statin doses. An e-health application optionally supported patient management. The primary outcome was change in lipid parameters over time. The secondary outcomes included evolocumab safety.

Results: Of 100 participants, 81\% had pre-existing CVD, $71 \%$ self-reported statin-related muscle symptoms, $44 \%$ received statins. All patients received evolocumab, 65\% were PCSK9i pre-treated at baseline. PCSK9i-naïve patients achieved a mean LDL-C reduction of $60 \%$ within 3 months of evolocumab treatment, which was maintained thereafter; $74 \%$ achieved LDL-C $<1.8 \mathrm{mmol} / \mathrm{L}$ at least once during observation, $69 \%$ attained $<1.4 \mathrm{mmol} /$

C. Mueller

Department of Cardiology, Cardiovascular Research Institute Basel (CRIB), University Hospital Basel, University of Basel, Basel, Switzerland

C. Crljenica $\cdot$ M. Rossi

Cardiocentro, Lugano, Switzerland

N. Reichert

Medical Affairs, Amgen Switzerland AG, Rotkreuz, Switzerland

I. Sudano

University Hospital Zurich, University Heart Center, Cardiology, Zurich, Switzerland 
L. In PCSK9i pre-treated patients, LDL-C remained stable throughout; $79 \%$ and $74 \%$ attained $<1.8 \mathrm{mmol} / \mathrm{L}$ and $<1.4 \mathrm{mmol} / \mathrm{L}$, respectively, at least once. Goal attainment was higher with any combination of evolocumab, statin, and/or ezetimibe. Overall, 89\% self-reported full evolocumab adherence. Treatmentemergent adverse events (TEAE) were reported in $30 \%$ of patients, two serious TEAEs occurred in one patient; three patients discontinued evolocumab because of TEAEs.

Conclusion: In real-world clinical practice, evolocumab was mainly used in patients with statin intolerance and pre-existing CVD. In this population, adherence to evolocumab and low LDL-C levels were maintained over 1 year, with better LDL-C goal achievement in patients using evolocumab in combination with other lipid-lowering drugs. Safety of evolocumab was similar to that documented in randomized controlled trials.

Keywords: Evolocumab; Real-world evidence; Adherence; Reimbursement; LDL-C goals; Cardiovascular disease

\section{Key Summary points}

In Swiss clinical practice, evolocumab was mainly used in patients with high/very high cardiovascular risk with statinrelated muscle symptoms and cardiovascular disease.

In this real-life setting, adherence to evolocumab was maintained over 1 year and the majority attained guideline recommended low-density lipoprotein cholesterol (LDL-C) goals.

LDL-C goal attainment was higher in patients using evolocumab in combination with other lipid-lowering therapies, especially with regards to LDL-C $<1.4 \mathrm{mmol} / \mathrm{L}$.

Effectiveness and safety of evolocumab in real-world clinical practice were comparable to those found in randomized controlled trials.

\section{INTRODUCTION}

Lipid-lowering therapy (LLT) reduces the cardiovascular risk of patients with cardiovascular disease by decreasing blood levels of low-density lipoprotein cholesterol (LDL-C) [1]. Several clinical trials have demonstrated the efficacy of statins, ezetimibe, and proprotein convertase subtilisin/kexin type 9 (PCSK9) inhibitors to reduce the risk of recurrent cardiovascular events among patients following an acute coronary syndrome or pre-existing cardiovascular disease. Therefore, European and American guidelines for cardiovascular prevention recommend the systematic use of LLT in all patients with or at increased risk for cardiovascular disease [2-5]. Once initiated, the cardiovascular benefit of LLT can be estimated on the basis of the absolute decrease in LDL-C levels as well as on the duration of treatment. However, despite consistent expert recommendations, the actual use and long-term adherence to LLT remains poor. In Europe, only half of patients achieve 2016 European Society of Cardiology (ESC)/European Atherosclerosis Society (EAS) guideline [6] recommended risk-based goals for LDL-C $(<1.8 \mathrm{mmol} / \mathrm{L})$ when treated with LLT $[7,8]$. In Switzerland, more than $60 \%$ of patients with acute coronary syndromes did not achieve 2016 ESC/EAS guideline recommended LDL-C goals at 1 year [9-11]; projected to 2019 ESC/EAS guideline [12] goals, this rate would be even lower. Studies from real-world practice therefore suggest that combination LLT is required [7] to meet ESC/EAS and Swiss Working Group on Lipids and Atherosclerosis (AGLA) [13] LDL-C goals.

Reasons for suboptimal LLT use are multiple and include side effects, difficulties with compliance to long-term daily oral administration, or reimbursement limitations. In observational studies, muscle pain has been reported in $10-30 \%$ of patients using statins, with very limited 5-year continuous adherence [14]. Based on effectiveness, appropriateness, and economic efficiency analysis (Swiss Federal Office of Public Health [FOPH] criteria), reimbursement of PCSK9 inhibitors is limited in Switzerland [15]. Evolocumab is a PCSK9 inhibitor that 
further reduces LDL-C levels and cardiovascular risk when combined with statins among patients with cardiovascular disease [16]. Evolocumab has been administered subcutaneously every 2 weeks with a very good 5 -year safety profile in clinical trials [17]. Therefore, evolocumab may help improve long-term adherence to LLT, with or without combination with statins. However, little data exists on the effectiveness, safety, and long-term adherence to PCSK9 inhibitors in the real-life setting governed by reimbursement limitations. In a multicenter observational study in Switzerland, we aimed to study the effectiveness, safety, and adherence of evolocumab over 1 year.

\section{METHODS}

\section{Study Design}

ECARA (Explore Clinical Utility of Evolocumab in Combination with an e-Health System: Swiss Prospective Observational Study in Patients with clinical Atherosclerotic Cardiovascular Disease) was a multicenter, prospective observational study conducted in Switzerland. Planned observation was up to 12 months after study enrollment. Four visits were scheduled at day 1 (baseline) and month 3, 6, and 12 (Fig. S1 in the supplementary material). The study was conducted at eight sites across urban regions in Switzerland; three centers were academic, one was private, and four were public health centers. All sites had experience in treating patient with lipid disorders.

\section{Study Population}

Between July 2017 and January 2019, ECARA enrolled adult patients ( $\geq 18$ years) receiving or planned to receive evolocumab. The decision to prescribe evolocumab was made by the treating physician before enrollment on the basis of the patients' medical need and according to Swissmedic approved label (www.swissmedic.ch) and Swiss Atherosclerosis Association (AGLA) recommendations [18]. Patients had clinical atherosclerotic cardiovascular disease (ASCVD) or a high or very high cardiovascular risk. At initiation of evolocumab, patients were using maximally tolerated doses of statin, or no statin in cases of complete statin intolerance. The history of statin-related muscle symptoms was collected as per the investigators' discretion (see "Protocol-Independent Guiding Principles"). Patients enrolled in an interventional clinical trial or in the observational Characteristics of hyperlipidemic patients at initiation of evolocumab and treatment patterns (HEYMANS) study [8], or patients with known hypersensitivity to evolocumab were excluded. Patients agreed to perform four scheduled visits and provided written informed consent.

\section{Protocol-Independent Guiding Principles}

Although not specified in the study protocol, evolocumab prescription in Swiss real-world clinical practice was guided by the Swiss reimbursement criteria (Version 01 July 2017) [15] described in detail in the supplement. Evolocumab is reimbursed concomitant to a diet and in addition to a maximum tolerated dose of an intensified LDL-C-lowering therapy for the treatment of patients with very high cardiovascular risk as primary prevention in familial hypercholesterolemia and an LDL-C $>5 \mathrm{mmol} / \mathrm{L}$ ( $>4.5 \mathrm{mmol} / \mathrm{L}$ in the presence of risk factors) or as secondary prevention in patients with homozygous familial hypercholesterolemia or clinical ASCVD when LDL-C $>3.5 \mathrm{mmol} / \mathrm{L}$ $(>2.6 \mathrm{mmol} / \mathrm{L}$ in case of progressive clinical ASCVD). According to Swiss reimbursement criteria, statin intolerance is considered to be demonstrated when multiple statin regimens have led to myalgia or an increase in creatinine kinase of at least fivefold above the upper limit of normal or if a statin has been associated with severe hepatopathy. According to Swiss reimbursement regulations, evolocumab treatment may only be continued if the follow-up examination 6 months after the start of treatment shows that the LDL-C under maximum intensified LLT has been reduced by at least $40 \%$ versus the initial value or an LDL-C value of $<1.8 \mathrm{mmol} / \mathrm{L}$ has been achieved (excluding homozygous familial hypercholesterolemia). 


\section{e-Health Application}

Enrolled patients could voluntarily install the mHealthAlert app (Cystelcom Sistemas S.A.U., Madrid, Spain) on their private mobile phones anytime during study visits. Data were automatically transferred to the customized smartphone app from a point of care measurement device for cholesterol profile and blood sugar, as well as separate but connected blood pressure and body weight devices. The app was also able to support medication adherence and consultation visits by sending reminders to patients. Anonymized data from the mHealthAlert cloud were transferred automatically to the electronic case report form (eCRF) for further analysis.

\section{Clinical Outcomes}

The primary objective of this study was to describe lipid parameters in patients receiving evolocumab at baseline and over 12 months in an observational real-world setting. The primary outcome was measured as cholesterol serum levels (total cholesterol [TC], LDL-C, high-density lipoprotein cholesterol [HDL-C], triglycerides) in millimoles per liter over time. LDL-C goal attainment was assessed as attainment of LDL-C $<1.8 \mathrm{mmol} / \mathrm{L}$ and $<1.4 \mathrm{mmol} / \mathrm{L}$ at least once during the 12-month observation period. An LDL-C value $<1.8 \mathrm{mmol} / \mathrm{L}$ refers to the 2016 ESC/EAS guidelines and an LDL-C goal of $<1.4 \mathrm{mmol} / \mathrm{L}$ refers to the $2019 \mathrm{ESC} / \mathrm{EAS}$ LDL-C guidelines. AGLA guidelines used in Switzerland had similar recommendations for LDL-C goals among high-risk patients. Secondary objectives were to collect demographic information, cardiovascular event history, comorbidities, and cardiovascular risk factors at baseline, and evolocumab safety. Medication use and "quality of life 12-item short form" (QoL SF-12) questionnaire were documented at baseline and at each scheduled assessment.

The exploratory objective was to estimate the value of the e-health application to support patient management, measured as a percentage of patients with treatment reminder usage over time and recording of evolocumab self-administration after 3, 6, and 12 months of study. An ad hoc analysis was conducted to estimate adherence. At each attended visit, patients were asked to estimate the number of missed doses since the last visit. Adherence was then defined as the percentage of patients reporting no missed doses while receiving evolocumab.

\section{Adverse Events}

Data on patient incidence of treatment-emergent adverse events, serious adverse events, and adverse events leading to discontinuation of evolocumab was collected. Treatment-emergent adverse events were defined as events that occurred between the first dose of evolocumab and the end of study; it was the investigator's responsibility to evaluate whether an adverse event was related to evolocumab. Definitions of (serious) adverse events, adverse device effects, as well as grading definitions are provided in the supplement. The Medical Dictionary for Regulatory Activities (MedDRA) version 18.0 or later was used to code all adverse events.

\section{Statistical Analysis}

All data summaries were descriptive in nature. For categorical variables (including the primary outcome measure), the frequency and percentage, with 95\% confidence interval (CI), were provided. Summary statistics for continuous variables included the number of patients, mean, median, standard deviation or standard error, 25th percentile (Q1), 75th percentile (Q3), minimum and maximum, and 95\% confidence intervals (CI, by Wilson score). Analyses were summarized for all enrolled patients and for the subgroups of patients with or without PCSK9 inhibitor pre-treatment at baseline. Between group comparisons were purely descriptive with no formal testing for statistical significance. All available data collected during the observation period were used in the analysis. No imputation of missing data was performed. All data were stored in a Microsoft SQL Server database and then exported to the statistical package R 3.6.2 (R Project) for the analysis (R Foundation for Statistical Computing, Vienna, Austria. https://www.R-project.org/). 


\section{Ethics Statement}

This study was performed in accordance with the Helsinki Declaration of 1964 and its later amendments. The lead medical ethics committee of the canton of Zurich approved the study protocol in cooperation with the ethics committees of the participating centers. All study participants gave written informed consent to participate in the study. The study was registered in the Registry of all Projects in Switzerland (RAPS) under study number BASEC ID 2016-01808 (https://raps.swissethics.ch/).

\section{RESULTS}

\section{Study Population}

Data of 102 patients were registered in the eCRF, but two observations were excluded because of screening failure $(n=1)$ and doubled data entry $(n=1) ; 100$ patients were therefore analyzed (full analysis set). Two patients partially withdrew, declining to attend further visits but giving consent for their data to be used. The median (Q1, Q3) age was 61 years (53, $67)$. The majority of patients were male $(72 \%$, $n=72)$ and had coronary artery disease $(84 \%$, $n=84)$. Most patients $(81 \%, n=83)$ had a prior cardiovascular event and 56\% $(n=56)$ had at least two previous cardiovascular events. Three patients (3\%) had familial hypercholesterolemia as documented by the investigator, all without a history of cardiovascular disease. The median (Q1, Q3) baseline LDL-C level was $1.9(1.3,3.4)$ $\mathrm{mmol} / \mathrm{L}$. Most patients $(71 \%, n=70$ of 99 reports) had a history of self-reported statin-related muscle symptoms (Table 1).

\section{LDL-C Management Before Enrollment}

Before enrollment, $44 \%$ of patients $(n=44)$ received statins, $30 \%(n=30)$ received ezetimibe, and $65 \%(n=65)$ had already received a PCSK9 inhibitor (PCSK9 inhibitor pre-treated) and either switched to evolocumab from another PCSK9 inhibitor or continued using evolocumab after baseline. The remaining 35\%
( $n=35)$ had no PCSK9 inhibitor before baseline (PCSK9 inhibitor naïve) and were initiated with evolocumab at baseline. Of PCSK9 inhibitor pretreated patients, 9\% $(n=6)$ had previously received alirocumab $75 \mathrm{mg}$ once every 2 weeks $(\mathrm{Q} 2 \mathrm{~W}), 89 \%(n=58)$ had received evolocumab $140 \mathrm{mg}$ Q2W, and 2\% $(n=1)$ evolocumab $420 \mathrm{mg}$ once monthly (QM).

\section{LDL-C Management During Observation}

After baseline, all patients received evolocumab with or without concomitant lipid-lowering agents with $46 \%(n=46)$ receiving evolocumab only, 24\% $(n=24)$ receiving evolocumab plus a statin, $20 \%(n=20)$ receiving evolocumab plus ezetimibe, and $10 \%(n=10)$ receiving evolocumab plus statin and ezetimibe. The split of LLTs was slightly different in PCSK9 inhibitor-naïve and pre-treated patients with more patients receiving the triplet combination in the PCSK9 inhibitor-naïve group (Fig. 1). Overall, 89\% $(n=89)$ of patients self-reported full adherence to the planned administration schedule, i.e., they did not report having missed any doses during the 12 months of observation. The proportion of patients receiving statins remained stable over the course of the study, with approximately $40 \%$. Prescribed statin intensity, however, changed over time with slightly fewer patients receiving high-intensity statins in the second half of the 12-month observation period (Fig. 2).

The number of patients taking non-statin lipid-lowering agents remained stable, ranging between $30 \%(n=30)$ and $38 \%(n=38)$. Of these, ezetimibe was prescribed to $79 \%(n=30 /$ $38)$ at baseline, $82 \%$ of patients $(n=28 / 34)$ at month $3,79 \%(n=23 / 29)$ at month 6 , and $83 \%$ $(n=25 / 30)$ at month 12 (Fig. 2).

\section{LDL-C Levels over Time and LDL-C Goal Achievement}

Overall, $83 \%$ of patients $(n=83)$ attained an LDL-C value $<1.8 \mathrm{mmol} / \mathrm{L}$ (2016 ESC/EAS LDLC goal) and 68\% $(n=68)$ achieved the 2019 ESC/EAS LDL-C goal of $<1.4 \mathrm{mmol} / \mathrm{L}$ at least once during the observation period. Goal 
Table 1 Baseline characteristics of the study population

\begin{tabular}{lll}
\hline Median (Q1, Q3) age, years & $n=100$ & $61(53,67)$ \\
Sex, $n$ (\%) & $n=100$ & $72(72)$ \\
Men & & $28(28)$ \\
Women & $n=100$ & \\
Type of symptomatic ASCVD ${ }^{\mathrm{a}}$ & & $84(84)$ \\
Coronary artery disease, $n$ (\%) & & $19(19)$ \\
Cerebrovascular disease, $n$ (\%) & & $20(20)$ \\
Peripheral artery disease, $n$ (\%) & & $3.9(3.2,5.7)$ \\
Median (Q1, Q3) lipid levels, mmol/L & $n=96$ & $1.9(1.3,3.4)$ \\
Total cholesterol & $n=91$ & $1.3(1.0,1.5)$ \\
LDL-C & $n=99$ & $1.5(1.0,2.1)$ \\
HDL-C & $n=93$ & \\
Triglycerides & $n=100$ & $44(44)$ \\
Lipid-lowering medication & \\
Statins ${ }^{\mathrm{b}}, n$ (\%) & & $30(30)$ \\
Ezetimibe, $n$ (\%) & & $65(65)$ \\
PCSK9ic, $n$ (\%) & & $70(71)$ \\
History of statin-related muscle symptoms, $n(\%)$ & $n=99$ &
\end{tabular}

${ }^{\text {a }}$ Patients could have more than one ASCVD or lipid-lowering agent

${ }^{\mathrm{b}}$ Statin dose could be zero in patients with severe statin intolerance

'Six patients had received prior alirocumab therapy and switched to evolocumab prior to enrollment into ECARA

attainment was higher in patients receiving evolocumab in combination with any background therapy of statin and/or ezetimibe with $87 \%$ achieving $<1.8 \mathrm{mmol} / \mathrm{L}$ and $78 \%$ reaching $<1.4 \mathrm{mmol} / \mathrm{L}$ at least once (Fig. S2 in the supplementary material).

Patients pre-treated with PCSK9 inhibitors before baseline $(n=65)$ maintained their already achieved low LDL-C levels over time with a reduction of LDL-C compared to baseline of $0.1 \mathrm{mmol} / \mathrm{L}(95 \% \mathrm{CI} 0.1,0.4)$ at month 12 ; $88 \%(95 \% \mathrm{CI} 78 \%, 94 \% ; n=57)$ and $68 \%$ $(95 \%$ CI 56\%, 78\%; $n=44)$ attained the 2016 and 2019 ESC/EAS LDL-C goals, respectively. In patients initiating evolocumab at baseline $(n=35)$, the mean reduction of LDL-C was $2.1 \mathrm{mmol} / \mathrm{L}(95 \% \mathrm{CI} 1.7,2.6)$ in the first 3 months of treatment, corresponding to a $60 \%$ of LDL-C reduction (Fig. 1). Low LDL-C levels were maintained thereafter with median LDL-C levels of $1.4 \mathrm{mmol} / \mathrm{L}$ or below over the remaining observation period. The overall LDL-C reduction of the 12 months was $2.3 \mathrm{mmol} / \mathrm{L}$ (95\% CI 1.9, 2.8). The LDL-C goal of $<1.8 \mathrm{mmol} / \mathrm{L}$ was achieved at least once in $74 \%$ $(95 \%$ CI $58 \%, 86 \% ; n=26)$ and $69 \%(95 \% \mathrm{CI}$ $52 \%, \quad 81 \% ; \quad n=24) \quad$ achieved $<1.4 \mathrm{mmol} / \mathrm{L}$. Again, goal attainment was higher in patients receiving any form of combination therapy in both groups (Fig. 1). Although patient numbers were low in individual groups of LLT type, there was a trend that goal attainment was highest in patients receiving triplet combinations of evolocumab plus statin and ezetimibe (Fig. S3 in the supplementary material). 
PCSK9i naive patients ( $n=35$ )

PCSK9i pre-treated patients $(n=65)$
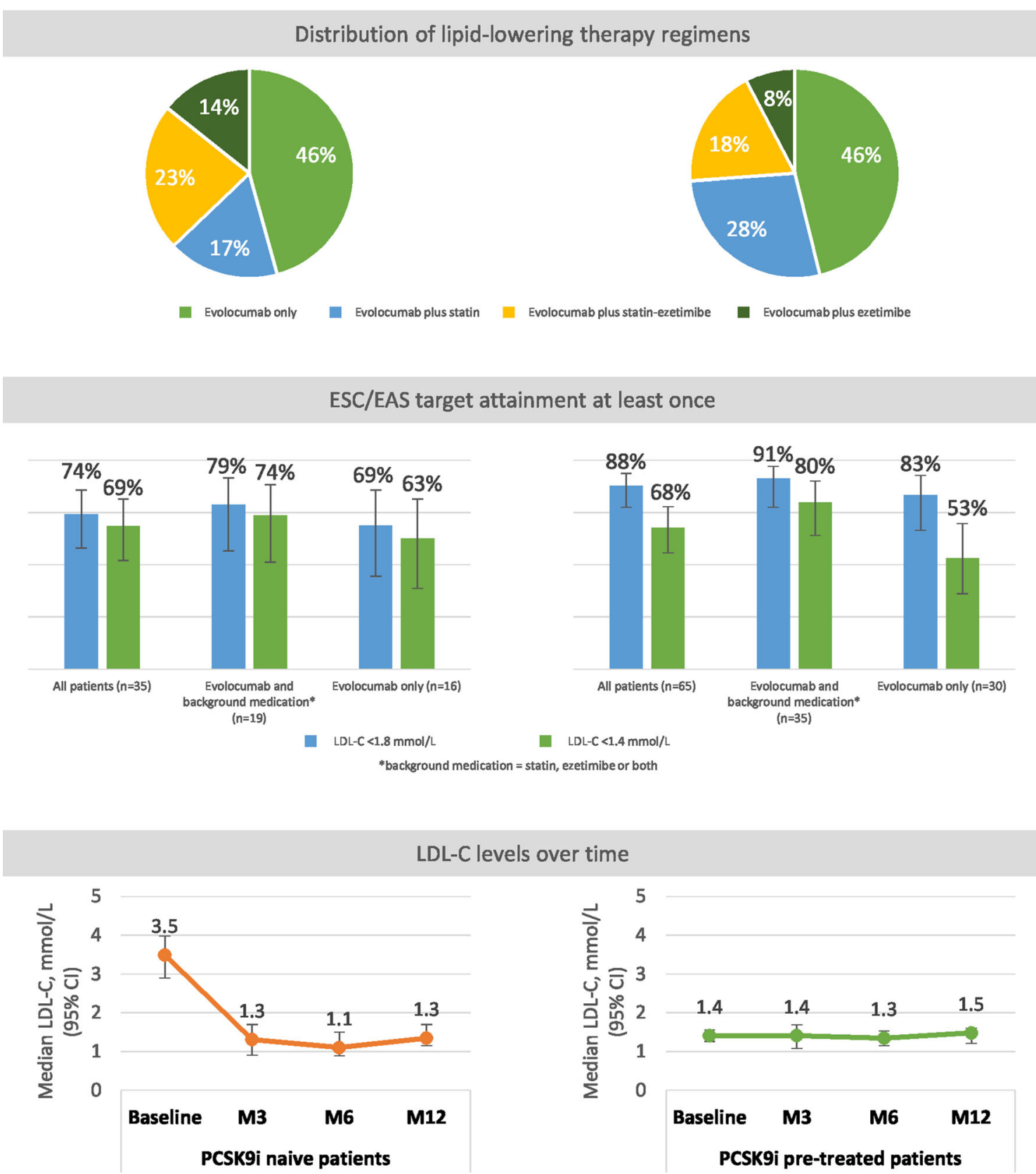

Fig. 1 Lipid-lowering therapies and treatment outcomes in PCSK9 inhibitor-naïve and pre-treated patients. CI confidence interval, EAS European Atherosclerosis Society, ESC European Society of Cardiology, M month, LDL-C

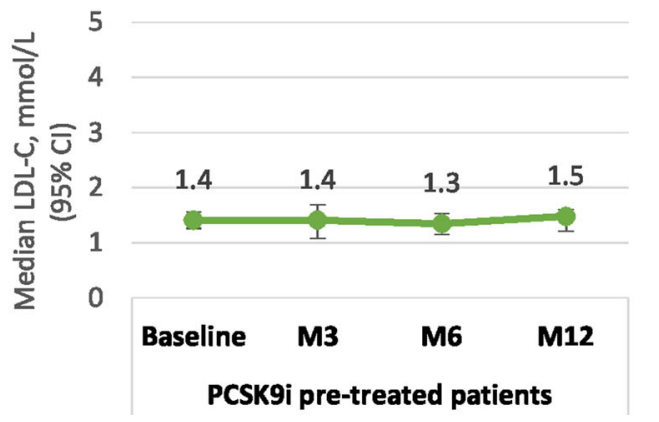

low-density lipoprotein cholesterol, PCSK9 proprotein convertase subtilisin/kexin type 9, PCSK9i PCSK9 inhibitor 


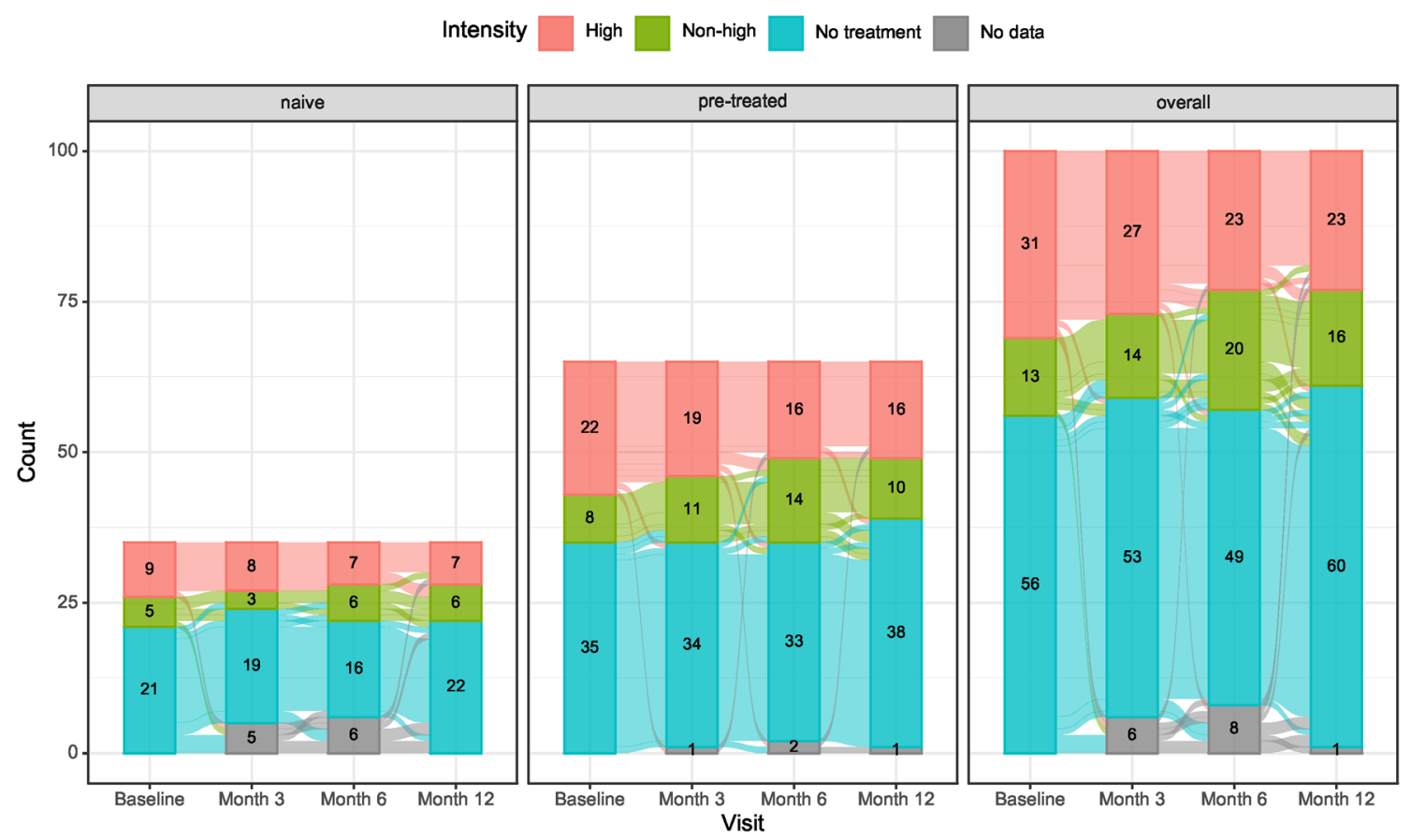

Fig. 2 Change in the number of patients receiving statin therapy over time, by statin intensity and PCSK9i status. Statin intensity, defined as per the American College of Cardiology/American Heart Association definition [28]

\section{Quality of Life}

Quality of life was not affected, and mental and physical health component scores remained stable between baseline and month 12 (Fig. S4 in the supplementary material).

\section{Safety}

Overall, 30 patients experienced a total of 78 adverse events. Seventy-six of these events were non-serious and 56\% ( $n=44$ events) were of mild severity, $36 \% \quad(n=28$ events $)$ were of moderate severity, and 5\% ( $n=4$ events) were severe. The two serious adverse events (increased blood creatine phosphokinase and myopathy) were of moderate severity and occurred in one patient. Three patients discontinued because of adverse events evolocumab (Table 2; Tables S1-S4 in the supplementary material).

\section{mHealthAlert App Usage}

Of the 100 patients in the study, 61\% $(n=61)$ used the mHealthAlert at least once. Of these, $66 \%(n=40)$ recorded at least one evolocumab self-administration over the 12-month study period, and $62 \%(n=38)$ used the treatment reminder at least once (Table S5 in the supplementary material). The exploratory analysis of LDL-C over time by intensity of usage of the app showed a trend towards LDL-C decline only in the group of heavy users $(n=15)$.

\section{DISCUSSION}

Consistent with the evolocumab randomized controlled clinical trial evidence (PROFICIO program), the present observational real-world study confirmed the LDL-C lowering effectiveness, safety and tolerability of evolocumab. Furthermore, this study suggests that effective adherence to evolocumab was maintained over 1 year. The mHealthAlert app, designed to 
Table 2 Treatment-emergent adverse events

\begin{tabular}{lll}
\hline Type of adverse event & $\boldsymbol{N}_{\text {events }}$ & $\boldsymbol{N}_{\text {patients }}$ \\
\hline Any adverse event, $n$ & 78 & 30 \\
Non-serious adverse events, $n^{\mathrm{a}}$ & 76 & $20(67)$ \\
Mild severity, $n$ (\%) & $44(56)$ & $17(57)$ \\
Moderate severity, $n(\%)$ & $28(36)$ & $2(7)$ \\
Severe, $n$ (\%) & $4(5)$ & 1 \\
Serious adverse event, $n^{\mathrm{a}}$ & 2 & $0(0)$ \\
Mild severity, $n$ (\%) & $0(0)$ & $1(3)$ \\
Moderate severity, $n$ (\%) & $2(3)$ & $0(0)$ \\
Severe, $n$ (\%) & $0(0)$ & 3 \\
Adverse event leading to withdrawal of product & 9 & \\
\hline
\end{tabular}

${ }^{\text {a }}$ The percentage of patients in the respective severity subgroups are based on the total number of adverse events $(n=78)$ and patients with adverse events $(n=30)$, respectively

Patients might experience more than one occurrence of the same event, more than one event in the same group, and also events in more than one group

enhance and monitor adherence, was only used more than once by a minority of patients.

This study was set up as a prospective, multicenter, observational study, observing patients treated with evolocumab as part of routine clinical management in Switzerland. The decision to prescribe evolocumab was made by the physician before enrollment and was guided by local reimbursement criteria after April 2017 (details see supplement). Swiss reimbursement conditions are set following three guiding principles: effectiveness, appropriateness and economic efficiency, and evolocumab prescription is thus limited to patients with very high cardiovascular risk concomitant to a diet and in addition to a maximum tolerated dose of an intensified LDL-C lowering therapy. As expected under these provisions, this study included a population of high- to very high-risk patients with significant comorbidities and recurrent cardiovascular disease.

Despite the well-known benefits of LDL-C lowering, a substantial proportion of patients in Switzerland with high- or very high cardiovascular risk was shown to receive suboptimal or no LLT [19]. In this observational study fewer than half (44\%) used statins at baseline, and $70 \%$ had a reported history of statin intolerance. Recent real-world cohorts reported a $50-80 \%$ rate of statin intolerance or inadequate statin dose due to intolerance of further dose escalation in patients referred for PCSK9 inhibitors [8, 20-22]. In this study, statin intolerance was documented as per the assessment of the treating physician. In Swiss clinical practice, the definition provided by the Swiss reimbursement criteria is commonly applied, which requires stringent clinical confirmation of myalgia or severe creatinine kinase elevation, following multiple statin regimens.

Accounting for the high rate of statin intolerance in this study population, more than half of patients received statin-free regimens with $46 \%$ of patients receiving evolocumab alone and $10 \%$ receiving evolocumab in combination with ezetimibe. Regardless of the regimen received, patients were able to achieve and maintain low LDL-C levels over time, suggesting adequate adherence to and long-term effectiveness of evolocumab-based regimens. Patients previously not treated with PCSK9 inhibitors achieved a $60 \%$ mean reduction on 
LDL-C (minus $2.1 \mathrm{mmol} / \mathrm{L}$ ) within the first 3 months of treatment. This is in line with a US real-world cohort in which a median LDL-C reduction of $60 \%$ was observed in patients receiving either evolocumab or alirocumab for 1 year [20]. A German cohort of patients receiving a PCSK9 inhibitor (evolocumab or alirocumab) achieved a reduction of LDL-C levels by $54 \%$ [22]. A Dutch cohort consisting of two-thirds of patients with familial hypercholesterolemia achieved a mean LDL-C reduction of $55 \%$ [23].

In this study, the LDL-C goal of $<1.8 \mathrm{mmol} /$ $\mathrm{L}$ was achieved at least once during observation by the majority of patients with $83 \%$ irrespective of the subgroup; two-thirds of the patients (68\%) reached the 2019 ESC/EAS LDL-C goal of $<1.4 \mathrm{mmol} / \mathrm{L}$. In the real-life setting, where evolocumab prescription is predominantly guided by reimbursement and limited to patients with partial or complete statin intolerance, 2019 ESC/EAS LDL-C goals are often not achieved, despite very good self-reported adherence (89\%) to PCSK9 inhibitor therapy.

Patients receiving evolocumab in combination with statins \pm ezetimibe or ezetimibe alone were more likely to achieve the recommended LDL-C goals, over three quarters of patients (78\%) attained LDL-C values $<1.4 \mathrm{mmol} / \mathrm{L}$. These findings are in line with previously published observational studies, which mostly show that recommended LDL-C values are often not achieved in daily clinical practice. Recently, the large DA VINCI observational study of realworld clinical practice in Europe [7] found a $54 \%$ achievement of the risk-based 2016 ESC/ EAS goal and 33\% achievement of the risk-based 2019 ESC/EAS goal. The LDL-C goal of $<1.8 \mathrm{mmol} / \mathrm{L}$ was achieved by $42 \%$ of patients in the previously mentioned German cohort [22], by $62 \%$ of patients in the Dutch cohort [23], and by $26 \%$ of patients in a retrospective health insurance database analysis of US patients [24]. In Switzerland, studies showed that two-thirds of post-ACS patients did not reach the ESC/EAS 2016 goal of $<1.8 \mathrm{mmol} / \mathrm{L}$, [9] in line with EUROASPIRE $\mathrm{V}$, where $71 \%$ of patients had LDL-C levels $\geq 1.8 \mathrm{mmol} / \mathrm{L}$ [25].

Interestingly, we found that patients pretreated with PCSK9 inhibitors received triplet combinations less frequently (18\%) than patients naïve to PCSK9 inhibitors at baseline (23\%). Although achievement of LDL-C levels $<1.8 \mathrm{mmol} / \mathrm{L}$ was lower among PCSK9 inhibitor-naïve patients, almost all patients achieving $<1.8 \mathrm{mmol} / \mathrm{L}$ also achieved $<1.4 \mathrm{mmol} / \mathrm{L}$; median LDL-C was slightly lower throughout the study in this group compared to PCSK9 inhibitor pre-treated patients. In the latter group, fewer patients achieving $<1.8 \mathrm{mmol} / \mathrm{L}$ also achieved $<1.4 \mathrm{mmol} / \mathrm{L}$ and although LDL-C was stable over time, it tended to be slightly higher than in the PCSK9 inhibitor-naïve group. An exploratory analysis of LDL-C goal attainment by individual LLT type showed a trend towards better attainment in patients receiving the triplet of evolocumab, statin, and ezetimibe. It needs to be noted that any analysis of goal attainment by LLT type did not take into consideration the statin intensity. The general analysis of statin intensity over time showed a decrease in the proportion of patients receiving high intensity statins over time from approximately three-quarters of administered statins being of high intensity at baseline to approximately half at months 6 and 12 .

The relatively low rate of LDL-C goal achievement in this study was possibly due to statin-free treatment in more than half of all patients (due to reported statin intolerance), insufficient statin intensity, or insufficient usage of combination therapy. As a result of narrow reimbursement regulations, real-world cohorts of PCSK9 inhibitor-treated patients have on average a very high proportion of statin-intolerant patients and the LDL-C threshold for evolocumab initiation set to very high levels in the Swiss reimbursement rules in 2017. Initiation thresholds were lowered to $>2.6 \mathrm{mmol} /$ L in secondary prevention in 2019 [15]. Considering the patient care pathway, low goal achievement may also indicate inadequate lipid management across assigned clinicians in routine practice in high- and very high-risk patients, as observed in a Swiss post-MI health care analysis describing medication possession and prescription behavior [26]. In addition, this was strikingly demonstrated by the DA VINCI study, where only $28 \%$ of patients were 
prescribed high-intensity statin monotherapy, $9 \%$ an ezetimibe combination, and $1 \%$ a PCSK9 inhibitor combination [7]. The new 2019 ESC/ EAS guidelines recommend an even more stringent LDL-C goal of $<1.4 \mathrm{mmol} / \mathrm{L}$ and extrapolations of current clinical practice to these new goals are sobering. As recently suggested by Stock, as many as 18 million patients with ASCVD in Europe will not reach these new goals [27]. Implementation of 2019 guidelines will thus require a practice change, especially among very high-risk patients and utilization of combination therapy $[8,27]$.

In the present study, $30 \%$ of patients experienced adverse events and three patients discontinued evolocumab because of adverse events. No analysis for a causal relationship with evolocumab treatment was conducted. This rate was substantially lower than in the randomized controlled FOURIER trial [3], where adverse events were documented in $77 \%$ of patients. Considering potential causality, in FOURIER only $1.6 \%$ of patients experienced adverse events leading to discontinuation of evolocumab.

The ECARA study has some limitations. As a result of its observational nature, treatment was documented as per clinical practice and adherence to treatment was only assessed by self-reporting of missed doses. Since the conduct of ECARA, new LDL-C goals have been defined by the ESC/EAS and the exploratory analysis of the present cohort with regards to the achievement of these new goals does not reflect that clinical practice had not yet implemented the new recommendations. Future studies will have to measure attainment of the new goals in a clinical setting that has had time to implement the new guidelines and also integrate the lessons learnt from studies such as ECARA, DA VINCI, and others that have notably shown a need for improvement of lipid management standards in order to reduce the cardiovascular risk.

\section{CONCLUSIONS}

This Swiss observational study confirms in realworld clinical practice previous findings from randomized controlled trials regarding effectiveness, adherence, and safety of evolocumab in a population at high/very high cardiovascular risk and a preponderance of statin intolerance. Using evolocumab, the patients were able to effectively and persistently lower LDL-C levels over 1 year, allowing two-thirds of patients to reach 2019 ESC/EAS and 2020 AGLA guidelines goals at least once, despite fewer than half receiving statin therapy. Goal achievement was highest in patients receiving combination therapy of evolocumab plus statins and ezetimibe. In current clinical practice, LDL-C goals are often not achieved, putting patients at risk of further cardiovascular events. The implementation of 2019 ESC/EAS guidelines with even more stringent LDL-C goals will likely require a practice change towards increased utilization of combination of lipidlowering therapy, including PCSK9 inhibitors, especially among very high-risk patients.

\section{ACKNOWLEDGEMENTS}

Funding. This study was funded by Amgen Switzerland AG. The study sponsor also funded the journal's rapid service and open access fee.

Authorship. All named authors meet the International Committee of Medical Journal Editors (ICMJE) criteria for authorship for this article, take responsibility for the integrity of the work as a whole, and have given their approval for this version to be published.

Author Contributions. David Nanchen, David Carballo, Isabella Sudano, and Nina Reichert contributed to the conception or design of the work. David Nanchen, David Carballo, Stefan Bilz, Hans Rickli, Konstantinos C. Koskinas, François Mach, Christian Mueller, Carmela Crljenica, Mariagrazia Rossi, and Isabella Sudano contributed to the acquisition of data for the work. All authors contributed to the analysis and interpretation of data for the work. David Nanchen, David Carballo, Nina Reichert, and Isabella Sudano drafted the manuscript. All authors critically revised the manuscript. All gave final approval and agree to be 
accountable for all aspects of work ensuring integrity and accuracy.

Medical Writing, Editorial and Other Assistance. Medical writing and editing assistance, including submission, were provided by Margit Hemetsberger, Hemetsberger medical services, Vienna, Austria, and was funded by Amgen Switzerland AG. The listed authors have authorized the submission of their manuscript via third party and approved any statements or declarations concerning conflicting interests, author contributions, funding, acknowledgements, and data sharing. Statistical analysis was provided by Adrian Olszewski, 2KMM Sp. z o.o., Katowice, Poland, and was funded by Amgen Switzerland AG.

Disclosures. David Carballo, Hans Rickli, François Mach, Carmela Crljenica, and Mariagrazia Rossi have nothing to disclose. David Nanchen is or has been investigator for clinical studies sponsored by Amgen, Pfizer, Daiichi Sankyo, Novartis. He has not received any personal fees in cash or in kind from these health industries. Stefan Bilz has received advisory board and speaker honoraria from Amgen, Sanofi, NovoNordisk, and AstraZeneca. Konstantinos C. Koskinas has received consulting fees and/or honoraria from Amgen and Daiichi Sankyo. Christian Mueller has received research support from the Swiss National Science Foundation, the Swiss Heart Foundation, the KTI, the European Union, the University of Basel, the University Hospital Basel, Abbott, Beckman Coulter, Idorsia, Ortho Cinical Diagnostics, Quidel, Roche, Siemens, Singulex, Sphingotec, as well as speaker honoraria/consulting honoraria from Acon, Amgen, Astra Zeneca, Bayer Boehringer Ingelheim, BMS, Idorsia, Novartis, Osler, Roche, and Sanofi outside of the submitted work. Nina Reichert is a full-time employee of Amgen Switzerland AG and holds Amgen stock options. Isabella Sudano reports consulting fees, travel grant and honoraria from: Amgen, AstraZeneca, Daiichi Sankyo, Medtronic, MSD, Recordati, Sanofi and Servier.

Compliance with Ethics Guidelines. This study was performed in accordance with the
Helsinki Declaration of 1964 and its later amendments. The lead medical ethics committee of the canton of Zurich approved the study protocol in cooperation with the ethics committees of the participating centers. All study participants gave written informed consent to participate in the study. The study was registered in the Registry of all Projects in Switzerland (RAPS) under study number BASEC ID 2016-01808 (https://raps.swissethics.ch/).

Data Availability. Qualified researchers may request data from Amgen clinical studies. Complete details are available at the following: https://wwwext.amgen.com/about/how-weoperate/policies-practices-and-disclosures/ ethical-research/clinical-data-transparencypractices/clinical-trial-data-sharing-request.

Open Access. This article is licensed under a Creative Commons Attribution-NonCommercial 4.0 International License, which permits any non-commercial use, sharing, adaptation, distribution and reproduction in any medium or format, as long as you give appropriate credit to the original author(s) and the source, provide a link to the Creative Commons licence, and indicate if changes were made. The images or other third party material in this article are included in the article's Creative Commons licence, unless indicated otherwise in a credit line to the material. If material is not included in the article's Creative Commons licence and your intended use is not permitted by statutory regulation or exceeds the permitted use, you will need to obtain permission directly from the copyright holder. To view a copy of this licence, visit http://creativecommons.org/licenses/by$\mathrm{nc} / 4.0 /$.

\section{REFERENCES}

1. Michos ED, McEvoy JW, Blumenthal RS. Lipid management for the prevention of atherosclerotic cardiovascular disease. N Engl J Med. 2019;381(16): 1557-67.

2. Giugliano RP, Cannon CP, Blazing MA, et al. Benefit of adding ezetimibe to statin therapy on 
cardiovascular outcomes and safety in patients with versus without diabetes mellitus. Circulation. 2018;137(15):1571-82.

3. Sabatine MS, Giugliano RP, Keech AC, et al. Evolocumab and clinical outcomes in patients with cardiovascular disease. N Engl J Med. 2017;376(18): 1713-22.

4. Schwartz GG, Steg PG, Szarek M, et al. Alirocumab and cardiovascular outcomes after acute coronary syndrome. N Engl J Med. 2018;379(22):2097-107.

5. Cholesterol Treatment Trialists' Collaboration, Baigent C, Blackwell L, et al. Efficacy and safety of more intensive lowering of LDL cholesterol: a metaanalysis of data from 170,000 participants in 26 randomised trials. Lancet. 2010;376(9753): 1670-81.

6. Catapano AL, Graham I, De Backer G, et al. 2016 ESC/EAS guidelines for the management of dyslipidaemias. Eur Heart J. 2016;37(39):2999-3058.

7. Ray KK, Molemans B, Schoonen WM, et al. EU-wide cross-sectional observational study of lipid-modifying therapy use in secondary and primary care: the DA VINCI study. Eur J Prevent Cardiol. 2021;28: 1279-89.

8. Ray KK, Bruckert E, Van Hout B, Feudjo Tepie M, Bridges I, Sibartie M. Does evolocumab use in Europe match 2019 ESC/EAS lipid guidelines? Results from the HEYMANS study. In: ESC Congress 2020. Virtual, 2020. Eur Heart J. 2020;41(Suppl. 2): ehaa946.3013. https://doi.org/10.1093/ehjci/ ehaa946.3013.

9. Gencer B, Koskinas KC, Raber L, et al. Eligibility for PCSK9 inhibitors according to American College of Cardiology (ACC) and European Society of Cardiology/European Atherosclerosis Society (ESC/EAS) guidelines after acute coronary syndromes. J Am Heart Assoc. 2017;6(11): e006537.

10. Koskinas KC, Gencer B, Nanchen D, et al. Eligibility for PCSK9 inhibitors based on the 2019 ESC/EAS and 2018 ACC/AHA guidelines. Eur J Prev Cardiol. 2021;28:59-65.

11. Rachamin Y, Meier R, Rosemann T, Langenegger S, Markun S. Statin treatment and LDL target value achievement in Swiss general practice-a retrospective observational study. Swiss Med Wkly. 2020;150:w20244.

12. Mach F, Baigent C, Catapano AL, et al. 2019 ESC/ EAS Guidelines for the management of dyslipidaemias: lipid modification to reduce cardiovascular risk. Eur Heart J. 2020;41(1):111-88.
13. Riesen WF, Kaiser W, Gallino A, von Eckardstein A, Theus G-R, Beer JH. Neue ESC/EAS-Dyslipidämieguidelines. Eine kommentierte Übersicht der AGLA. Swiss Med Forum. 2020;20(09-10):140-8.

14. Vonbank A, Agewall S, Kjeldsen KP, et al. Comprehensive efforts to increase adherence to statin therapy. Eur Heart J. 2017;38(32):2473-9.

15. Swiss Federal Office of Public Health. Specialties list. https://www.bag.admin.ch/dam/bag/en/ dokumente/kuv-leistungen/arzneimittel/Spezialit\% C3\%A4tenliste/spezialitaetenliste-als-pdf-standvom-01-02-2020.pdf.download.pdf/Spezialit\%C3\% A4tenliste\%20als\%20PDF\%20Stand\%20vom\% 2001.02.2020.pdf. Accessed Nov 13, 2020.

16. Sabatine MS, Giugliano RP, Wiviott SD, et al. Openlabel study of long-term evaluation against LDLCI. Efficacy and safety of evolocumab in reducing lipids and cardiovascular events. N Engl J Med. 2015;372(16):1500-9.

17. Koren MJ, Sabatine MS, Giugliano RP, et al. Longterm efficacy and safety of evolocumab in patients with hypercholesterolemia. J Am Coll Cardiol. 2019;74(17):2132-46.

18. von Eckardstein A, Riesen WF, Carballo D, et al. Empfehlungen zur Prävention der Atherosklerose 2018: update der AGLA. Swiss Med Forum. 2018;18(47):975-80.

19. Gencer B, Carballo D, Nanchen D, et al. Intensified lipid lowering using ezetimibe after publication of the IMPROVE-IT trial: a contemporary analysis from the SPUM-ACS cohort. Int J Cardiol. 2020;303: 8-13.

20. Kaufman TM, Warden BA, Minnier J, et al. Application of PCSK9 inhibitors in practice. Circ Res. 2019;124(1):32-7.

21. Zafrir B, Jubran A. Lipid-lowering therapy with PCSK9-inhibitors in the real-world setting: two-year experience of a regional lipid clinic. Cardiovasc Ther. 2018;36(5): e12439.

22. Saborowski M, Dölle $M$, Manns MP, Leitolf $H$, Zender S. Lipid-lowering therapy with PCSK9-inhibitors in the management of cardiovascular highrisk patients: effectiveness, therapy adherence and safety in a real world cohort. Cardiol J. 2018;25(1): $32-41$.

23. Stoekenbroek RM, Hartgers ML, Rutte R, de Wijer DD, Stroes ESG, Hovingh GK. PCSK9 inhibitors in clinical practice: delivering on the promise? Atherosclerosis. 2018;270:205-10.

24. Menzin J, Aggarwal J, Boatman B, et al. Ezetimibe use and LDL-C goal achievement: a retrospective 
database analysis of patients with clinical atherosclerotic cardiovascular disease or probable heterozygous familial hypercholesterolemia. J Manag Care Spec Pharm. 2017;23(12):1270-6.

25. De Backer G, Jankowski P, Kotseva K, et al. Management of dyslipidaemia in patients with coronary heart disease: results from the ESC-EORP EUROASPIRE V survey in 27 countries. Atherosclerosis. 2019;285:135-46.

26. Huber CA, Meyer MR, Steffel J, Blozik E, Reich O, Rosemann T. Post-myocardial infarction (MI) care: medication adherence for secondary prevention after MI in a large real-world population. Clin Ther. 2019;41(1):107-17.

27. Stock JK. DA VINCI study: change in approach to cholesterol management will be needed to reduce the implementation gap between guidelines and clinical practice in Europe. Atherosclerosis. 2020;314:74-6.

28. Grundy SM, Stone NJ. 2018 American Heart Association/American College of Cardiology multisociety guideline on the management of blood cholesterol: primary prevention. JAMA Cardiol. 2019;4(5):488-9. 